\title{
Acupuncture in Seasonal Allergic Rhinitis (ACUSAR) - Design and Protocol of a Randomised Controlled Multi-Centre Trial
}

\author{
Benno Brinkhaus ${ }^{\mathrm{a}}$ Claudia M. Witt ${ }^{\mathrm{a}}$ Miriam Ortiz $^{\mathrm{a}}$ Stephanie Roll ${ }^{\mathrm{a}}$ Thomas Reinhold ${ }^{\mathrm{a}}$ \\ Klaus Linde ${ }^{\mathrm{b}}$ Florian Pfab ${ }^{\mathrm{c}, \mathrm{d}}$ Bodo Niggemann ${ }^{\mathrm{e}}$ Josef Hummelsberger ${ }^{\mathrm{f}}$ Dominik Irnich ${ }^{\mathrm{g}}$ \\ Karl Wegscheider ${ }^{\mathrm{h}}$ Stefan N. Willich ${ }^{\mathrm{a}}$ \\ ${ }^{a}$ Institute for Social Medicine, Epidemiology, and Health Economics, Charité - University Medical Center, Berlin, \\ ${ }^{b}$ Institute of General Practice, Klinikum rechts der Isar, Technische Universität München, Munich, \\ ${ }^{c}$ Department of Dermatology and Allergy, Technische Universität München, Munich, \\ ${ }^{\mathrm{d}}$ Division of Environmental Dermatology and Allergy, Helmholtz Zentrum München/TUM, ZAUM-Center for Allergy and Environment, Munich, \\ e Pediatric Allergology and Pneumology, German Red Cross Hospital Westend, Berlin, \\ ${ }^{\mathrm{f}}$ International Society for Chinese Medicine (Societas Medicinae Sinensis, SMS), Munich, \\ ${ }^{9}$ Department of Anaesthesiology, Ludwig-Maximilians-University, Munich, \\ ${ }^{\mathrm{h}}$ Department of Medical Biometry and Epidemiology, University Medical Center Eppendorf, Hamburg, Germany
}

\section{Key Words}

Acupuncture · Traditional Chinese medicine .

Complementary and alternative medicine - Allergy .

Seasonal allergic rhinitis · Randomised controlled trial

\section{Summary}

Background: We report on the study design and protocol of a randomised controlled trial (Acupuncture in Seasonal Allergic $R$ hinitis, ACUSAR) that investigates the efficacy of acupuncture in the treatment of seasonal allergic rhinitis (SAR). Objective: To investigate whether acupuncture is non-inferior or superior to (a) penetrating sham acupuncture and (b) rescue medication in the treatment of SAR. Design: 3-armed, randomised controlled multi-centre trial with a total follow-up time of 16 weeks in the 1st year and 8 weeks in the 2 nd year. Setting: 41 physicians in 37 out-patient units in Germany specialised in acupuncture treatment. Patients: 400 seasonal allergic rhinitis patients with clinical symptoms and test-positive (skin-prick test and/or specific $\lg$ E) to both birch and grass pollen. Interventions: Patients will be randomised in a 2:1:1 ratio to one of three groups: (a) semi-standardised acupuncture plus rescue medication (cetirizine); (b) penetrating sham acupuncture at non-acupuncture points plus rescue medication; or (c) rescue medication alone for 8 weeks (standard treatment group). Acupuncture and sham acupuncture will consist of 12 treatments per patient over 8 weeks. Main Outcome Measures: Average means of the Rhinitis Quality of Life Questionnaire (RQLQ) overall score and the Rescue Medication Score (RMS) between weeks 6 and 8 in the first year, adjusted for baseline values. Outlook: The results of this trial available in 2011 will have a major impact on the decision of whether acupuncture should be considered as a therapeutic option in the treatment of SAR.

\author{
Schlüsselwörter \\ Akupunktur - Traditionelle chinesische Medizin . \\ Komplementärmedizin · Allergie · Saisonale \\ allergische Rhinitis · Randomisierte klinische Studie
}

\section{Zusammenfassung}

Hintergrund: Wir berichten über das Studiendesign und essenzielle Teile des Protokolls einer randomisierten Studie (Acupuncture in Seasonal Allergic Rhinitis, ACUSAR) bei Patienten mit saisonaler allergischer Rhinitis. Ziel: Ziel der Studie ist es, zu untersuchen, ob Akupunktur in der Therapie von saisonaler allergischer Rhinitis nicht unterlegen bzw. wirksamer ist als (a) penetrierende Sham-Akupunktur bzw. (b) Bedarfsmedikation allein. Design: Dreiarmige kontrollierte klinische MulticenterStudie mit einem Follow-up von 16 Wochen im 1. und 8 Wochen im 2. Jahr. Prüfzentren: 41 auf Akupunktur spezialisierte Ärzte in 39 Praxen und Ambulanzen in Deutschland. Patienten: 400 Patienten mit klinisch manifester und positiv getesteter (Prick-Test und/oder spezifisches IgE) saisonaler allergischer Rhinitis auf Birken- und Gräserpollen. Studienintervention: $\mathrm{Pa}$ tienten erhalten nach einer 2:1:1-Verteilung randomisiert entweder (a) eine semi-standardisierte Akupunkturbehandlung mit Bedarfsmedikation (Cetirizin), (b) eine penetrierende ShamAkupunktur an Nicht-Akupunktur-Punkten mit Bedarfsmedikation oder (c) Bedarfsmedikation alleine für 8 Wochen. Die Akupunktur-Intervention erfolgt in 12 Akupunktursitzungen über einen Zeitraum von 8 Wochen. Hauptzielkriterien: Jeweils die Score-Mittelwerte des "Rhinitis Quality of Life Questionnaire» (RQLQ) und der Bedarfsmedikation gemessen mit dem "Rescue Medication Score" (RMS) zwischen Woche 6 und 8, adjustiert für die Baseline-Werte. Ausblick: Die Studienergebnisse sind 2011 zu erwarten und werden die Basis für eine wissenschaftliche Neubeurteilung der Akupunktur bei saisonaler allergischer Rhinitis bieten.

\section{KARGER}

Fax +497614520714

Information@Karger.de

www.karger.com (c) 2010 S. Karger GmbH, Freiburg

Accessible online at:

www.karger.com/fok

Charité - University Medical Center, Berlin

10098 Berlin, Germany

Tel. +49 30450 529-002, Fax -902

benno.brinkhaus@charite.de 


\section{Introduction}

Allergic rhinitis (AR) has become a major health problem. In the past 2 decades, there has been a marked increase in the prevalence of AR [1]. Direct yearly costs for AR in Europe are estimated at 1.0-1.5 billion Euros annually, whereas indirect costs are estimated at 1.0-2.0 billion Euros [1]. A remarkable number of patients are turning for relief to complementary and alternative medicine (CAM), such as acupuncture. The lifetime prevalence of CAM use in patients with AR ranges from $27-46 \%$, and most of the patients who have not yet used CAM intend to do so in the future $[2,3]$. In particular, acupuncture is used by $17-19 \%$ of AR patients [2, 3]. Because of this, acupuncture has increasingly become the focus of allergy specialists and scientists $[4,5]$. In addition, acupuncture was judged by specialists $(48 \%)$ to be the CAM treatment best supported by experimental evidence [4].

However, studies on the efficacy of acupuncture in patients with AR have yielded inconclusive results [6-12]. For example, results of our pilot study indicate that the use of a combination of acupuncture and Chinese herbal medicine is superior to sham acupuncture and placebo treatment in patients with seasonal allergic rhinitis (SAR) [9]. Furthermore, results of a large pragmatic trial with $>5,000$ patients, published recently, suggested that treating patients who suffer from AR with adjunctive acupuncture therapy leads to clinically relevant benefits [12]. In addition, Xue et al. and $\mathrm{Ng}$ et al. [8, 11] demonstrated that acupuncture is effective in reducing subjective AR symptom scores while Magnusson et al. [10] found that acupuncture was not superior to sham acupuncture in reducing clinical symptoms. The results of our prior trials $[9,12]$ provided the background and basis of the ACUSAR ( puncture in Seasonal Allergic Rhinitis) trial (fig. 1).
Authors of two recently published systematic reviews draw the conclusion that there is currently insufficient evidence to support or refuse the use of acupuncture in patients with AR $[13,14]$. They pointed out that previous studies on the efficacy of acupuncture in AR have suffered from a variety of methodological limitations, such as small patient numbers or the lack of a sham-acupuncture control group and they stated, that a large well-conducted RCT, which overcomes identified methodological problems would be required.

\section{Aim of the Study}

The primary objective of the ACUSAR trial is to investigate whether an 8-week semi-standardised acupuncture plus rescue medication is non-inferior (1st step) or superior (2nd step) to (a) penetrating sham acupuncture intervention plus rescue medication and (b) rescue medication alone in the treatment of SAR. Secondary objectives include an assessment of long-term effectiveness and use of rescue medication over a period of 8 weeks in the 2 nd year and an evaluation of cost-effectiveness of acupuncture versus standard treatment.

\section{Patients and Methods}

\section{Design}

The ACUSAR study is a 3-armed, randomised, parallel controlled multicentre trial investigating the efficacy of acupuncture plus rescue medication versus penetrating sham acupuncture (involving superficial needling at non-acupuncture points) plus rescue medication in patients with SAR as well as the efficacy of acupuncture plus rescue medication versus rescue medication alone (fig. 2). Patients are blinded to treatment concerning acupuncture and penetrating sham acupuncture in the study. After randomisation, patients in the acupuncture and penetrating sham acupuncture groups receive 12 treatment sessions over a period of 8 weeks.
Fig. 1. ACUSAR study and previously conducted studies on the effectiveness of acupuncture in patients with AR. CHM = Chinese herbal medicine; RCT $=$ randomised controlled clinical trial.

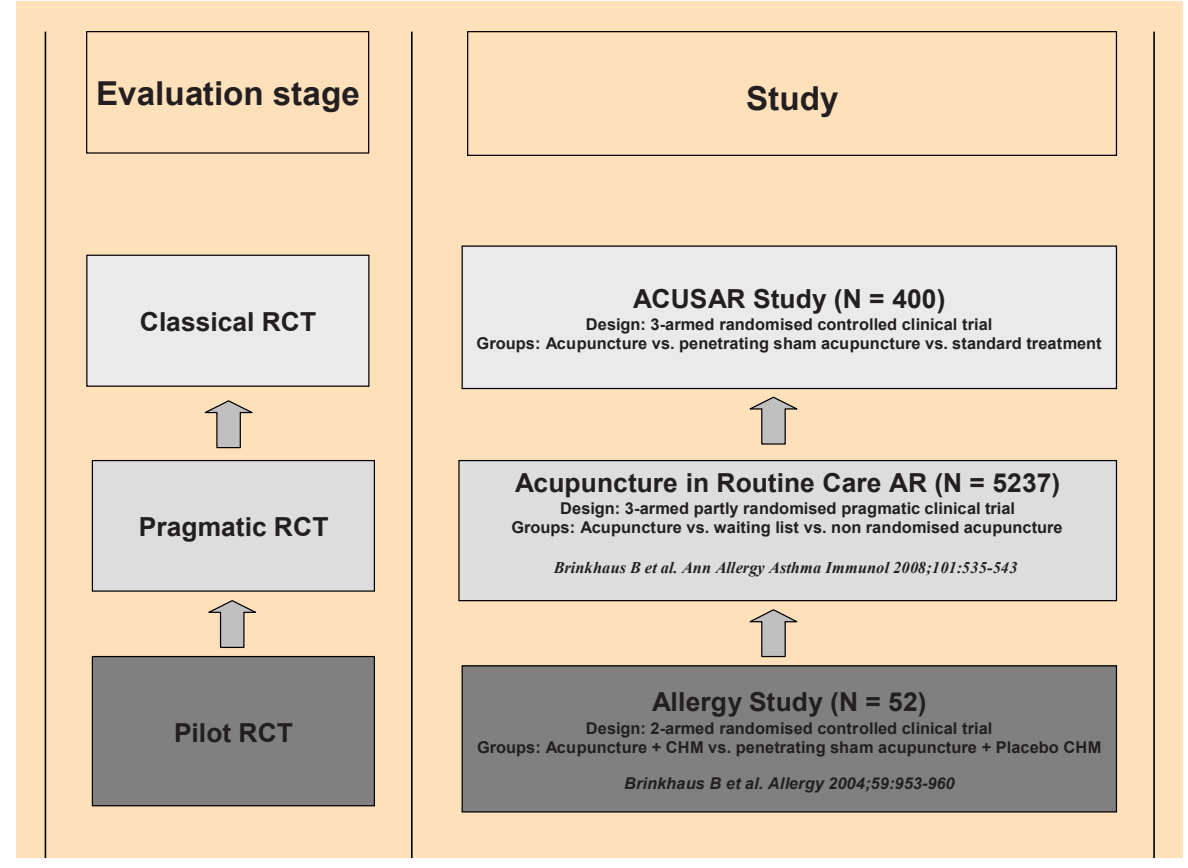


Fig. 2. Trial design, time schedule, and outcome parameters of the ACUSAR study.). Outcome measures: RQLQ = Rhinitis Quality of Life Questionnaire; RMS = amount of antiallergic medication assessed daily by the Rescue Medication Score; VAS = visual analogue scale of SAR symptoms; SF-36 = German version of the health-related quality of life questionnaire.

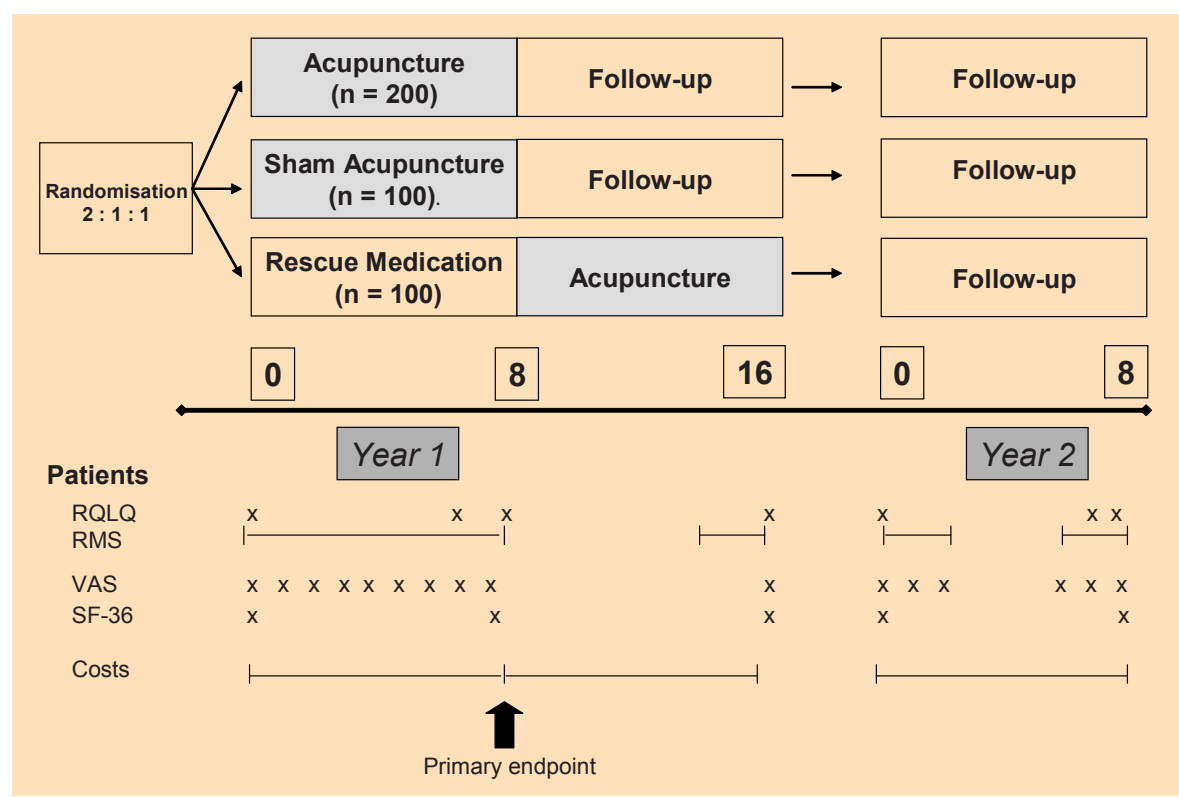

In the rescue medication group, all patients receive rescue medication only for the first 8 weeks. During the following 8 weeks, they receive 12 acupuncture treatments in the same way as in the group treated with a semi-standardised acupuncture regimen. The total follow-up study period per patient is 16 weeks in the 1 st year and 8 weeks in the 2 nd year.

This study is conducted according to common guidelines for clinical trials (Declaration of Helsinki, Version von Somerset West 1996), including certification by an external in-house audit according to ICH-GCP at the Institute for Social Medicine, Epidemiology, and Health Economics, Charité University Medical Center Berlin, Germany, after recruitment of $50-80 \%$ of the target sample size. The study protocol has been approved by the ethics review of the Charite University Hospital Berlin.

For concealment of the allocation of the next patient, individual patient randomisation (2:1:1) into the 3 study arms is performed centrally by the external clinical trial unit (Koordinationszentrum Klinische Studien, KKS) at the Charite University Hospital Berlin. Patients who meet the inclusion criteria and give written and oral consent are included in the study. After a patient is included in the study, his or her physician phones the KKS, where the patient will be registered. Subsequently, the physician receives information from the KKS regarding patient allocation both by phone and fax.

\section{Patients}

The ACUSAR trial aims to recruit a total of 400 patients. Recruitment for the trials started in April 2008. For inclusion in the ACUSAR study, patients must meet the following criteria: female or male patients (aged $16-45$ years) with SAR, clinically and test-positive (skin-prick test and/or specific IgE - at least class 2 of the radio-allergo-sorbent test (RAST), results to grass and birch pollen; patients with $>2$ years of moderate to severe SAR; visual analogue scale $>40$ and $<80 \mathrm{~mm}$ for SAR symptoms during the past year; patients must be able to complete a diary for selfevaluation of symptoms and recording use of anti-symptomatic medication; use of, or indication for, oral antihistamines as antiallergic medication, and written informed consent.

Main exclusion criteria for patients are one or more of the following diseases or conditions: perennial SAR or other types of chronic rhinitis; allergic asthma and/or moderate to severe atopic dermatitis; auto-immune disorders; severe chronic inflammatory diseases; history of anaphylactic reactions; hypersensitivity to rescue medication (cetirizine) or related drugs used in the study; specific immunotherapy for $>3$ years; simul- taneous participation in other clinical trials; serious acute or chronic organic disease or mental disorder; pregnancy or breast feeding; current allergy desensitisation therapy, or during the past 2 years, or planned in the next 2 years. Further exclusion criteria include blood coagulation disorder and/or current use of anticoagulants; previous acupuncture treatment for SAR; any CAM treatment at the moment, in the last 3 months, or planned within the 2 trial years.

\section{Participating Physicians}

Participating trial physicians were recruited from a network of approximately 100 practices; this network was established during our previous randomised acupuncture trials [15-18]. Physicians were required to fulfill all of the following criteria: (1) acupuncture training at least equivalent to an 'A-diploma' from one of the major German acupuncture societies (140 $\mathrm{h}$ of acupuncture training); (2) $\geq 3$ years of practical experience with acupuncture; (3) participation in study training sessions on the trial methods, the interventions tested, and standards for performing clinical trials (ICH-GCP); (4) $50 \%$ of trial physicians had to have at least a 'B-diploma' $(350 \mathrm{~h}) ;(5) 50 \%$ had to have experience working in clinical studies. 41 practitioners in 37 out-patient units in Germany are currently participating in the studies. Non-medical acupuncturists were not included in the study.

\section{Study Intervention and Informed Consent}

The treatment strategies for acupuncture and penetrating sham acupuncture were developed in a consensus process with experienced acupuncture experts (see Acknowledgement) from two major German societies for medical acupuncture: the German Medical Acupuncture Association (Deutsche Ärztegesellschaft für Akupunktur; DÄGfA), Munich, and the International Society for Chinese Medicine, Munich (Societas Medicinae Sinensis, SMS). The acupuncture and the penetrating sham acupuncture were developed using the Delphi method with three rounds (two meetings and one writing round) including acupuncture experts from two major acupuncture societies and experts on trial methodology. The final strategies were generally considered as a pragmatic compromise between the need for standardization and for individualization.

Patient randomisation and the study intervention start at the beginning of the birch pollen season. Both the acupuncture and the penetrating sham acupuncture treatments consist of 12 sessions of $\geq 20$ and $\leq 40 \mathrm{~min}$ duration each, administered over a period of 8 weeks (preferably 2 ses- 
Table 1. Acupuncture points used in the ACUSAR study

\begin{tabular}{|c|c|c|}
\hline Category of points & Points & Selection \\
\hline $\begin{array}{l}\text { Basic acupuncture points } \\
\text { (obligatory) }\end{array}$ & $\begin{array}{l}\text { L.I. } 4 \\
\text { L.I. } 11 \\
\text { L.I. } 20 \\
\text { EX-HN } 3 \text { Yintang }\end{array}$ & $\begin{array}{l}\text { bilateral (except Yintang), } \\
\text { altogether } 7 \text { points }\end{array}$ \\
\hline $\begin{array}{l}\text { Basic acupuncture points } \\
\text { (facultative) }\end{array}$ & $\begin{array}{l}\text { EX-HN } 8 \text { BITONG } \\
\text { GB } 20 \\
\text { LIV } 3 \\
\text { LU } 7 \\
\text { ST } 36 \\
\text { SP } 6 \\
\text { SJ } 17 \\
\text { BL } 13\end{array}$ & uni- or bilateral, $\geq 3$ points \\
\hline $\begin{array}{l}\text { Additional local acupuncture points } \\
\text { (facultative) }\end{array}$ & $\begin{array}{l}\text { BL } 2 \\
\text { GB } 1,14 \\
\text { EX-HN } 5 \text { TAIYANG } \\
\text { SI } 18 \\
\text { SJ } 23 \\
\text { ST } 2 \\
\text { further points }\end{array}$ & $\begin{array}{l}\geq 3 \text { points (local and } \\
\text { distant additional points) } \\
\text { uni- or bilateral }\end{array}$ \\
\hline $\begin{array}{l}\text { Additional distant acupuncture points } \\
\text { (facultative) }\end{array}$ & $\begin{array}{l}\text { LU } 1,5 \\
\text { Ma } 44 \\
\text { GB } 41,34,37 \\
\text { LIV 2 } \\
\text { LIV 5 } \\
\text { KID 3, } 7 \\
\text { BL 12, 20, 23, 26, } 40 \\
\text { SP 9 } \\
\text { REN 6, 17, 22, } 20 \\
\text { SJ 5, } 6 \\
\text { further points }\end{array}$ & \\
\hline $\begin{array}{l}\text { Additional ear acupuncture } \\
\text { (facultative) }\end{array}$ & $\begin{array}{l}\text { Allergy point } \\
\text { Shenmen point } \\
\text { Thymus point } \\
\text { ACTH point } \\
\text { further points }\end{array}$ & \\
\hline
\end{tabular}

sions in each of the first 4 weeks, followed by 1 session per week in the remaining 4 weeks). Patients in the rescue medication group do not receive acupuncture treatment for a period of 8 weeks. After 8 weeks, they also receive the acupuncture treatment described below (waiting list design). In all three groups moxibustion is not allowed.

Acupuncture treatment in the ACUSAR study is semi-standardised (table 1): All patients have to be treated at 4 obligatory basic acupuncture points: LI 4, LI 11, LI 20 bilaterally, and Ex-HN 3 (Yintang). Furthermore, at least 3 out of 8 facultative basic acupuncture points have to be selected according to the principles of traditional Chinese medicine (TCM). In addition, patients have to be treated at $\geq 3$ local and/or distant acupuncture points. Acupuncturists may use additional points including ear acupuncture points. The number and name of all acupuncture points used in each session must be documented. A traditional Chinese syndrome diagnosis is mandatory and the diagnosis must be documented. Needle length and diameter are not predefined but have to be documented. An irradiating needling sensation ('de qi') should be achieved, if possible. Needles should be stimulated manually at least once in each session.

Number, duration, and frequency of the sessions in the penetrating sham acupuncture group are the same as in the acupuncture group. Par- ticipating trial physicians are required to choose $\geq 5$ points for penetrating sham acupuncture treatment out of a selection of 7 penetrating sham acupuncture points at non-classical sites which have to be needled bilaterally ( $\geq 10$ needles altogether) in each session (table 2). Superficial insertion only using fine needles ( $\leq 20 \mathrm{~mm}$ long) is recommended. Needling sensation 'de qi' and manual stimulation of the needles should be avoided; the needles should be placed subcutaneously. All acupuncturists were trained to apply penetrating sham acupuncture and received a DVD showing detailed information on how to perform the penetrating sham acupuncture.

Patients of all three groups are allowed to take $\leq 2$ doses of secondgeneration oral antihistamines daily (e.g., $2 \times 1$ cetirizine dihydrochloride/ day), if required. If the clinical symptom(s) of SAR cannot be sufficiently stabilised by the antihistamine treatment, an oral steroid can be used additionally. The patients are instructed to precisely document the applied rescue medication on a daily basis in a special diary.

Patients will be instructed not to use any of the following medications or treatments during the study period in both years: topical cromolyns (eye drops and nasal spray), topical antihistamines, topical steroids, leukotriene receptor antagonists, anti-cholinergic agents, $\alpha$-adrenergic agonists, allergen immunotherapy, nasal ipratropium, decongestants, and any form of CAM for SAR. 
Table 2. Penetrating sham acupuncture points used in the ACUSAR study

\begin{tabular}{|c|c|c|}
\hline $\begin{array}{l}\text { Name of sham } \\
\text { acupuncture points }\end{array}$ & Areas & Selection \\
\hline Deltoideus & $\begin{array}{l}\text { In the middle of the insertion line of M. deltoideus (L.I. 14) and } \\
\text { Acromion (between L.I. and SJ meridian) }\end{array}$ & \multirow{7}{*}{$\begin{array}{l}5 \text { out of } 7 \text { proposed } \\
\text { points should be needled } \\
\text { bilaterally (in total } 10 \\
\text { points) }\end{array}$} \\
\hline Upper arm & In the mid line between the acupuncture points LU 3 and L.I.14 & \\
\hline Upper leg I & $\begin{array}{l}6 \text { cun above the upper edge of the patella (between the spleen } \\
\text { and stomach meridian) }\end{array}$ & \\
\hline Upper leg II & $\begin{array}{l}5 \text { cun above the upper edge of the patella (between the spleen } \\
\text { and stomach meridian) }\end{array}$ & \\
\hline Upper leg III & $\begin{array}{l}4 \text { cun above the upper edge of the patella (between the spleen } \\
\text { and stomach meridian) }\end{array}$ & \\
\hline Back I & 5 cun laterally of the spine of thoracal vertebrum 8 & \\
\hline Back II & 5 cun laterally of the spine of lumbar vertebrum 1 & \\
\hline
\end{tabular}

In order to minimise bias from patients' expectations, patients are informed in the same way as in the in the ART trials [15-18]: 'In this study, different types of acupuncture will be compared. One type is similar to the acupuncture treatment used in China. The other type does not follow these principles, but has also been associated with positive outcomes in clinical studies.'

\section{Outcome Measurement}

For the evaluation of the main and secondary outcome measures, patients are requested to fill in a questionnaire including the Rhinitis Quality of Life Questionnaire (RQLQ) and the symptom scores (global and subscores) before randomisation and at the end of weeks 7, 8, and 16 in the 1 st year and at the beginning of the birch pollen season as well as at the end of week 8 in the following year (fig. 2). In addition, patients are requested to fill in diaries including the Rescue Medication Score (RMS) in the first 8 weeks and in the weeks 14-16 in the 1st year and in the weeks $1-2$ and 6-8 in the 2 nd year.

Main outcome measures in the ACUSAR study are average means of the RQLQ overall score and the RMS between weeks 6 and 8 in the 1st year, adjusted for baseline values.

The RQLQ is a well-established and validated questionnaire created by Juniper et al. [19,20] and has been used in several clinical studies in conventional medicine and also in complementary medicine e.g., in acupuncture $[9,12]$ as a primary or secondary outcome measure. The RQLQ consists of an overall score and 7 sub-scores including limitations of activities, sleep problems, non-hay fever symptoms, practical problems, nasal symptoms, eye symptoms, and emotions. Rescue medication usage will be scored daily using the RMS [21] on a 4-point scale as follows: no rhinitis medication ( 0 points); oral antihistamines $-1 \times$ cetirizine dihydrochloride $10 \mathrm{mg} /$ day or equivalent (1 point); oral antihistamines $-2 \times$ cetirizine dihydrochloride $10 \mathrm{mg} /$ day ( $=20 \mathrm{mg}$ ) or equivalent ( 2 points); any form of systemic steroids or other drug for SAR (3 points).

Secondary outcome measures include the RQLQ score at week 16 in the 1st year, and at the end of week 7 and 8 in the 2nd year; the RMS in week 1 and between weeks 15 and 16 in the 1st year and between weeks 6 and 8 during the pollen season in the 2nd year; responders to study intervention are defined as patients with a change in RQLQ score of $\geq 0.5$ between the baseline RQLQ score and the mean of the RQLQ scores from weeks 6 to 8; a Visual Analogue Scale (VAS, 0-100 mm) SAR overall symptom severity and the VAS nasal, eye, pharyngeal, and common symptoms weekly between baseline and week 8 , at week 17 and 18 in the 1st year, and at weeks 1 and 2, and weeks 6 and 8 in the 2nd year and the two summary scales (physical and mental health) of the health-related quality of life SF-36 [22, 23].

Further secondary outcome parameters are: (1) the 'Havelhöher Konstitutionsfragebogen' (T-HKF, Version 2.4) questionnaire [24] to assess patients' constitution, (2) global assessment of change in week 8 by the patient measured by the questionnaire on overall treatment effect at week 8 after randomisation $(0=$ worsening, $1=$ no change, $2=$ slight to moderate improvement, 3 = good to excellent improvement); (3) level of cost-effectiveness as a result of health economic analyses from a society's perspective, and (4) the number of side-effects and serious and non-serious adverse events.

In order to test the effectiveness of blinding, patients in the acupuncture and sham acupuncture groups fill in a questionnaire after the $3 \mathrm{rd} \mathrm{ac}-$ upuncture session in order to assess the credibility of the respective treatment methods [25]. At the end of the study, patients in these two groups are asked whether they believe that they have received acupuncture following the principles of Chinese medicine or the other type of acupuncture. For each session, physicians are asked to report whether side-effects or adverse or serious adverse events effects occurred. In addition, the patients are asked to report side-effects in the abovementioned questionnaires (see fig. 1). Drop-outs, withdrawals, and the respective reasons are documented.

\section{Data Monitoring and Safety Board}

The Data Monitoring and Safety Board (DMSB) ensures the safety of the trial participants, the credibility of the study, and the validity and integrity of the data. Early in the trial, the DMSB reviews focus on safety, quality of conduct, and trial integrity. Interim data reports are submitted every other month during the pollen season. Each month during the recruitment phase in the 2 study years, the DSMB receive a report of side-effects and any serious adverse events. The DMSB consists of 3 experts independent of the study team: a statistician, an expert for research in CAM, and an acupuncturist.

\section{Statistical Analysis}

The primary outcome measures are the RQLQ and the RMS between weeks 6 and 8 .

Analyses will be performed for 2 populations: (1) an intention-to-treat (ITT) population with all patients randomised and treated with at least 1 acupuncture session; for the primary analysis, missing values will be replaced by multiple imputations, and (2) a per-protocol population including only patients with no major protocol deviations. First, all available data will be analysed descriptively. Results will be summarised as means, standard deviations, and 95\%-confidence intervals (CIs) for continuous data; medians, quartiles, and ranges for rank data; and percentages for discrete data.

Rescue medication use and quality of life are linked in a complicated manner: a higher amount of rescue medication may or may not improve quality of life independent of the direct acupuncture effects; on the other hand, a reduced quality of life may induce a more intensive use of 
rescue medication. Thus, it is not sufficient to prove superiority in one of the two endpoints if non-inferiority cannot be proven for the other endpoint. Correspondingly, for each of the two two-group comparisons, non-inferiority has to be proven first for both of the primary outcome parameters before superiority in at least one of them is worthwhile to be studied. In more detail, for confirmatory testing, an analysis of covariance of the primary outcome measure will be performed in the ITT group (1) on the RQLQ between weeks 6 and 8, adjusting for the RQLQ score at baseline, and (2) on the RMS between weeks 6 and 8 , adjusting for the RMS score at baseline. For primary analysis, the test procedure will be hierarchical (1-sided tests, $\alpha=0.025)$ : (1) In a first step, acupuncture will be compared to penetrating sham acupuncture group using a test of non-inferiority with respect to RQLQ. The non-inferiority test is significant if the left-sided ANCOVA-based $97.5 \%$ CI of the between-group difference lies completely above the non-inferiority margin of -0.5 . (2) In the case of significance in step 1, in a 2 nd step, acupuncture will be compared to the penetrating sham acupuncture group using a test of non-inferiority with respect to RMS. The non-inferiority test is significant if the left-sided ANCOVA-based 97.5\% CI of the between-group difference lies completely above the non-inferiority margin of -1.5 , chosen proportionally to the non-inferiority limit of the RQLQ. (3) In the case of significance in step 2, in a 3rd step, acupuncture will be compared to the sham acupuncture group with a test of superiority with respect to the RQLQ or the RMS. For this purpose, 2 left-sided ANCOVA-based $98.75 \%$ CIs of the between-group difference in RQLQ and RMS will be calculated and compared to zero. The test of superiority is significant if at least one of the two CIs lies completely above zero. Steps 4,5 , and 6 will be analogous to steps 1,2 , and 3 , but with the rescue medication group instead of the sham acupuncture group. We will perform exploratory analyses for all secondary outcomes as well as for the comparison of sham acupuncture group with the rescue medication group.

For the power calculation, we used the RQLQ data from our previous trial on the effectiveness of acupuncture [12]. As no previous data on RMS are available, the power calculation was performed only for the two hypotheses relating to the RQLQ. However, we assume that our sample size will be sufficient, as we expect that the effect sizes shown by acupuncture for the RMS are similar to those shown for the RQLQ. Based on the assumption of a non-inferiority limit of 0.5 (= limit of clinical difference; see [20] in a 1-sided test (significance level $\alpha=0.025$ ), a power of $80 \%$, and a common standard deviation of 1.1 (data from previous trial [12]), we used nQuery Advisor ${ }^{\circledR} 4.0$ to determine a sample size of 164 in the acupuncture group, 82 in the rescue medication control, and 82 in the penetrating sham acupuncture group using a 2:1:1 allocation ratio. Assuming a drop-out rate of approximately $20 \%$, we will require a sample size of 200 in the acupuncture group, 100 in the rescue treatment control, and 100 in the penetrating sham acupuncture group.

\section{Discussion}

To our knowledge, the ACUSAR study is the first clinical study to investigate the efficacy of an acupuncture treatment for SAR compared to both a penetrating sham acupuncture treatment and a standard treatment in a multi-centre 3-armed clinical trial. Compared to previous studies of acupuncture in the treatment of SAR including a sham acupuncture group, the ACUSAR study has a much larger number of participating acupuncturists, a more rigorous methodology and will include significantly more patients.

Because of the discontinuous appearance of symptoms in patients with SAR, the effectiveness of acupuncture is not easy to investigate in this disease. Therefore, we choose as inclusion criteria SAR testing positive to grass and birch pollen. In Germany, the birch pollen season usually lasts from early March to mid May and the grass pollen season from late April to mid August resulting in a SAR symptom phase of about 5 months for each patient annually. However, depending on the weather influence and the geographical region, the pollen flow is different in strength even in the birch and grass pollen season. We cannot control for each patient's SAR symptoms individually but we think that the randomisation process will balance the patients according to the different pollen flight and SAR symptoms in the three groups.

As the trial will be performed in a large number of private practices without special diagnostic equipment, the inclusion and exclusion criteria must be pragmatic. However, the diagnosis will have to be defined once by an AR specialist using a skin-prick test and/or RAST before study entry. The inclusion and exclusion criteria of the study are in accordance with those proposed by the Consensus Statement in Allergic Rhinitis [1]. The main inclusion criteria (allergy history of $>2$ years, positive prick test to grass and birch pollen, severity of symptoms), have been applied in other clinical studies evaluating the efficacy of conventional treatment $[26,27]$ as well as in our earlier acupuncture trial [9]. Our study is designed to ensure that patient characteristics, and thus also the results of this study, will be comparable to previous trials.

Acupuncture practiced according to the principles of Chinese medicine is an individualised therapy [28]. In the ACUSAR study, we use a semi-standardised treatment protocol, similar to a number of other studies in the past, including the 4 ART studies [15-18]. In all patients, we require that a number of pre-defined 'basic points' be used obligatory and facultative. In addition, physicians are allowed to add further points based, for example, on an individualised syndrome diagnosis made according to TCM diagnostic methods, or on clinical experience. As in the ART trials [15-18], the predefined 'basic points' were selected in a consensus process with experienced acupuncture experts while taking the principles of TCM into account. The main reason for choosing a semi-standardised treatment was to foster transparency and a certain degree of reproducibility without losing individualisation. As moxibustion is commonly not used to treat SAR, the decision to exclude this treatment which is associated with acupuncture was unproblematic. Many acupuncture studies published so far have been criticised for unclear or insufficient quality of acupuncture [29]. As in the ART trials [15-18], we have used explicit and rigid criteria to ensure that the physicians in the study have a high level of education and practical experience. Nevertheless, it must be pointed out that the education of German acupuncturists, also in this trial, varies considerably. All trial acupuncturists are physicians who have completed their primary training ( $>80 \%$ have $\geq 350$ h of training) in acupuncture in Germany and have spent little or no time training in China. 
A major issue in the planning phase of the ACUSAR study was the choice of a sham control group. The sponsor of this trial, the German research foundation, has explicitly requested that research be conducted on acupuncture compared with 'placebo,' or 'sham' acupuncture. However, the concepts of placebo and its specific, and unspecific effects in relation to complex physical interventions such as acupuncture are unclear (details see [30]). Various different forms of sham and placebo acupuncture were used in the past [31]. Both penetrating sham acupuncture and the placebo acupuncture with the sham acupuncture needle [32] touch the skin and therefore evoke activity of cutaneous afferent nerves [33]. We are aware that penetrating sham acupuncture is not a valid placebo intervention [33]. But as all other sham control concepts fail to represent a valid placebo as well, we use - as in the ART trials penetrating sham acupuncture as 'sham control group'.

For standard treatment we use oral second-generation antihistamines and - in the case of not sufficiently treated SAR symptoms - in addition oral steroids. However, the guideline treatment also includes topical oral antihistamines or steroids as primary or second-line treatment [1]. The reason to choose oral antihistamines in the rescue medication treatment was that oral antihistamines are still the most widely used SAR treatment and, in addition, the RMS can be calculated more easily.

In this study, we apply a range of outcome measures commonly used in AR research. Both primary outcome measures, the RQLQ overall score and the RMS are well established and of clear clinical relevance: The RQLQ is a well-validated questionnaire $[19,20]$ and has been used in several highquality studies as a primary outcome parameter [26, 34, 35]. A further advantage is that we have already used the RQLQ in our first two trials on the efficacy of acupuncture in AR $[9$, 12]. Therefore, we will be able to compare the results of this trial with those of the earlier ones. The documentation and analyses of the rescue medication is common in allergy trials $[36,37]$. However, it would be desirable to take into consideration more objective endpoints in acupuncture research. Therefore, we implemented two smaller experimental trials in two sub-groups of ACUSAR patients to study changes in immunology parameters (ACUSAR EXP) and vegetative functions (AUTO ACUSAR) triggered by acupuncture.

In conclusion, the ACUSAR study is one of the largest and most rigorous studies of acupuncture including a sham control group conducted in SAR thus far. This study will have an impact on the decision of whether acupuncture should be considered as a therapeutic option in the treatment of SAR.

\section{Acknowledgements and Conflict of Interest}

The ACUSAR study is funded by a grant of the German Research Foundation (Deutsche Forschungsgemeinschaft, DFG), Grant No. WI 957/161. Trial Register Number: ClinicalTrials.gov: NCT00610584.

We would like to thank members of the review board of the 'Deutsche Forschungsgemeinschaft' (DFG) for their helpful advice to the ACUSAR trial methodology, in particular Prof. Dr. Hans-Joachim Trampisch, Bochum, Dr. Armin Koch, Bonn, Prof. Dr. Eva Hummers-Pradier, Hannover, and Dr. Frank Wissing and the members of the DMSB: Prof. Dr. Ralf Kohnen, Prof. Dr. Andreas Michalsen, and Dr. Helmut Rüdinger.

The following acupuncture and methodological experts participated in the consensus process on study intervention: Prof. Dr. Benno Brinkhaus, Berlin, Dr. Jochen Gelditsch, Baierbrunn; Dr. Carl-Hermann Hempen, München; Dr. Josef Hummelsberger, München; PD Dr. Dominik Irnich, München; PD Dr. Klaus Linde, München; PD Dr. Florian Pfab, München; Prof. Dr. Claudia Witt, Berlin; Dr. Michael Wullinger, Rosenheim.

Conflicts of Interst: None declared.

\section{References}

1 Van Cauwenberge P, Bachert C, Passalacqua G, Bousquet J, Canonica GW, Durham SR, et al: Consensus statement on the treatment of allergic rhinitis. European Academy of Allergology and Clinical Immunology. Allergy 2000;55:116-134.

2 Krouse JH, Krouse HJ: Patient use of traditional and complementary therapies in treating rhinosinusitis before consulting an otolaryngologist. Laryngoscope 1999;109:1223-1227.

3 Schafer T, Riehle A, Wichmann HE, Ring J: Alternative medicine in allergies - prevalence, patterns of use, and costs. Allergy 2002:57:694-700.

4 Senna G, Passalacqua G, Bonadonna P, Agostinis F, Lombardi C, Cocco G, et al: Is alternative medicine acceptable in allergology? Allergy 2000;55:1200.

5 Bielory L: 'Complementary and alternative medicine' population based studies: a growing focus on allergy and asthma. Allergy 2002;57:655-658.

6 Xue CC, An X, Cheung TP, Da CC, Lenon GB, Thien FC, et al: Acupuncture for persistent allergic rhinitis: a randomised, sham-controlled trial. Med J Aust 2007;187:337-341.
7 Wolkenstein E, Horak F: Protective effect of acupuncture on allergen provoked rhinitis. Wien Med Wochenschr 1998;148:450-453.

8 Xue CC, English R, Zhang JJ, Da Costa C, Li CG: Effect of acupuncture in the treatment of seasonal allergic rhinitis: a randomized controlled clinical trial. Am J Chin Med 2002;30:1-11.

9 Brinkhaus B, Hummelsberger J, Kohnen R, Seufert $\mathrm{J}$, Hempen $\mathrm{CH}$, Leonhardy $\mathrm{H}$, et al: Acupuncture and Chinese herbal medicine in the treatment of patients with seasonal allergic rhinitis: a randomizedcontrolled clinical trial. Allergy 2004;59:953-960.

10 Magnusson AL, Svensson RE, Leirvik C, Gunnarsson RK: The effect of acupuncture on allergic rhinitis: a randomized controlled clinical trial. Am J Chin Med 2004;32:105-115.

$11 \mathrm{Ng}$ DK, Chow PY, Ming SP, Hong SH, Lau S, Tse $\mathrm{D}$, et al: A double-blind, randomized, placebocontrolled trial of acupuncture for the treatment of childhood persistent allergic rhinitis. Pediatrics 2004;114:1242-1247.
12 Brinkhaus B, Witt CM, Jena S, Liecker B, Wegscheider K, Willich SN: Acupuncture in patients with allergic rhinitis: a pragmatic randomized trial. Ann Allergy Asthma Immunol 2008;101:535-543.

13 Roberts J, Huissoon A, Dretzke J, Wang D, Hyde C: A systematic review of the clinical effectiveness of acupuncture for allergic rhinitis. BMC Complement Altern Med 2008;8:13.:13.

14 Lee MS, Pittler MH, Shin BC, Kim JI, Ernst E: Acupuncture for allergic rhinitis: a systematic review. Ann Allergy Asthma Immunol 2009;102:269-279.

15 Linde K, Streng A, Jürgens S, Hoppe A, Brinkhaus B, Witt C, et al: Acupuncture for patients with migraine - a randomized trial (ART Migraine). JAMA 2005;293:2118-2125.

16 Witt C, Brinkhaus B, Jena S, Linde K, Streng A, Wagenpfeil S, et al: Acupuncture in patients with osteoarthritis of the knee: a randomised trial. Lancet 2005;366:136-143.

17 Melchart D, Streng A, Hoppe A, Brinkhaus B, Witt C, Wagenpfeil S, et al: Acupuncture in patients with tension-type headache - a randomised trial. BMJ 2005;331:376-382. 
18 Brinkhaus B, Witt CM, Jena S, Linde K, Streng A, Wagenpfeil S, et al: Acupuncture in patients with chronic low back pain - a randomised controlled trial. Arch Intern Med 2006;166:450-457.

19 Juniper EF, Guyatt GH: Development and testing of a new measure of health status for clinical trials in rhinoconjunctivitis. Clin Exp Allergy 1991;21:77-83.

20 Juniper EF, Guyatt GH, Griffith LE, Ferrie PJ: Interpretation of rhinoconjunctivitis quality of life questionnaire data. J Allergy Clin Immunol 1996;98:843-845.

21 Kuehr J, Brauburger J, Zielen S, Schauer U, Kamin W, Von BA, et al: Efficacy of combination treatment with anti-IgE plus specific immunotherapy in polysensitized children and adolescents with seasonal allergic rhinitis. J Allergy Clin Immunol 2002;109:274-280.

22 Bousquet J, Bullinger M, Fayol C, Marquis P, Valentin B, Burtin B: Assessment of quality of life in patients with perennial allergic rhinitis with the French version of the SF-36 Health Status Questionnaire. J Allergy Clin Immunol 1994;94:182-188.

23 Bullinger M, Kirchberger I: SF-36 Fragebogen zum Gesundheitszustand. Göttingen, Hogrefe, 1998.

$24 \mathrm{Kroz}$ M, von Laue HB, Zerm R, Girke M: Development of a questionnaire for endogenous regulation - a contribution for salutogenesis research. Forsch Komplementarmed Klass Naturheilkd 2003;10:70-77.

25 Vincent C: Credibility assessments in trials of acupuncture. Complement Med Res 1990;4:8-11.

26 Bousquet J, Lund VJ, Van CP, Bremard-Oury C, Mounedji N, Stevens MT, et al: Implementation of guidelines for seasonal allergic rhinitis: a randomized controlled trial. Allergy 2003;58:733-741

27 Radcliffe MJ, Lewith GT, Turner RG, Prescott P, Church MK, Holgate ST: Enzyme potentiated desensitisation in treatment of seasonal allergic rhinitis: double blind randomised controlled study. BMJ 2003;327:251-254

28 Cheng X: Chinese Acupuncture and Moxibustion. Beijing, Foreign Languages Press, 1987.

29 Pomeranz B: Acupuncture analgesia - basic research; in Stux G, Hammerschlag R (eds): Clinical Acupuncture: Scientific Basis. Berlin, Springer, 2001, pp 1-28.

30 Melchart D, Linde K, Streng A, Reitmayr S, Hoppe A, Brinkhaus B, et al: Acupuncture randomized trials (ART) in patients with migraine or tension-type headache - design and protocols. Forsch Komplementärmed Klass Naturheilkd 2003;10:179-184.

31 Dincer F, Linde K: Sham interventions in randomized clinical trials of acupuncture - a review. Complement Ther Med 2003;11:235-242.

32 Streitberger K, Kleinhenz J: Introducing a placebo needle into acupuncture research. Lancet 1998;352:364-365.

33 Lund I, Naslund J, Lundeberg T: Minimal acupuncture is not a valid placebo control in randomised controlled trials of acupuncture: a physiologist's perspective. Chin Med 2009;4(1):1.

34 Noonan MJ, Raphael GD, Nayak A, Greos L, Olufade AO, Leidy NK, et al: The health-related quality of life effects of once-daily cetirizine $\mathrm{HCl}$ in patients with seasonal allergic rhinitis: a randomized double-blind, placebo-controlled trial Clin Exp Allergy 2003;33:351-358.

35 Kim LS, Riedlinger JE, Baldwin CM, Hilli L, Khalsa SV, Messer SA, et al: Treatment of seasonal allergic rhinitis using homeopathic preparation of common allergens in the southwest region of the US: a randomized, controlled clinical trial. Ann Pharmacother 2005;39:617-624

36 Casale TB, Condemi J, LaForce C, Nayak A, Rowe M, Watrous M, et al: Effect of omalizumab on symptoms of seasonal allergic rhinitis: a randomized controlled trial. JAMA 2001;286:2956-2967.

37 Durham SR, Walker SM, Varga EM, Jacobson MR, O'Brien F, Noble W, et al: Long-term clinical efficacy of grass-pollen immunotherapy. N Engl J Med 1999;341:468-475. 